\title{
El Instituto de Academias de Andalucía: Génesis y regulación legal
}

\section{Eduardo Roca Roca}

Arbor CLXIII, 641 (Mayo 1999), 19-36 pp.

En Anda'lucía ha existido una antigua tradición Académica paralela con la génesis y evolución de las Academias Centrales de Madrid, siendo creadas a lo largo de los siglos XVIII y XIX, y que recogen los movimientos culturales andaluces, a través de diversas Academias de diversa extensión territorial. Tras la Constitución de 1978, se aprueba el Estatuto de Autonomía'de Andalucía, asumiendo esta Comunidad en el art. 13 de su Estatuto, competencia exclusiva en materia de Academias con sede central en Andalucía. La necesidad de actuación coordinada de tales Academias dió lugar a la creación del Instituto de las Reales Academias de Andalucía por Ley 7/ 1985 de 6 de Diciembre, el cual como Corporación de Derecho Público integra a las Academias de Andalucía, las coordina entre si y con la Junta de Andalucía, a la vez, crea y refuerza vínculos con el Instituto de España, y otras Instituciones Administrativas y Culturales españolas y extranjeras.

\section{Antecedentes: Las Academias andaluzas}

En Andalucía existe una antigua tradición académica, que es paralela con la génesis y desenvolvimiento de las Academias centrales radicadas en Madrid, lo cual tiene una evidente justificación como consecuencia de la estructura geográfica y la división administrativa española durante los siglos XVIII y XIX. 


\section{Eduardo Roca Roca}

Las Academias centrales, radicadas en Madrid, responden al principio de centralización que domina durante los siglos XVIII y XIX, y la consiguiente radicación de las organizaciones culturales del País, como se desprende en la creación de las primeras Reales Academias por Felipe V y Fernando VI, a cuyo efecto se puede recordar la antiguedad de la Real Academia de Nobles Artes que más tarde se denominará de Bellas Artes de San Fernando, la Real Academia Española o la Real Academia de la Historia (Reales Cédulas de 12 de abril de 1752 y 30 de mayo de 1757) que tienen una finalidad específica respecto a la conservación de la lengua española, o la tutela de monumentos históricos y restos arqueológicos, siendo curioso destacar que a mediados del siglo XIX se crean diversas Academias Provinciales de Bellas Artes como Organos periféricos para la más inmediata tutela y protección de la propiedad monumental.

Sucesivamente van apareciendo las diversas Academias centrales en las que se van a cultivar distintos aspectos de las Letras o de las Ciencias (Medicina, Ciencias Exactas Físicas y Naturales, Ciencias Morales y Políticas, Jurisprudencia y Legislación, Farmacia, etc).

Las dificultades de comunicación que se producen, no sólo en el ámbito físico, sino tambien en el intelectual, entre el centro y la periferia, darán lugar al nacimiento de Instituciones Académicas similares en distintos sectores geográficos españoles, surgiendo de esta manera Academias que tienen carácter regional, provincial e, incluso, local en cuanto limitan sus actividades al Municipio en el que se establece su sede.

Andalucía no fué una excepción en este movimiento Académico, sino que se puede afirmar que el mismo se produce de forma paralela al de Madrid y, en algunos momentos, se anticipa a estos movimientos culturales de la Capital del Reino. Para confirmar lo dicho es suficiente recordar que la Real Academia de Medicina de Sevilla fué creada por el Rey Don Carlos II en el año 1700, por lo que puede afirmarse que es la Real Academia más antigua de España con un funcionamiento ininterrumpido desde su fundación. En la Andalucía Oriental tambien es destacable la antiguedad de la Real Academia de Jurisprudencia y Legislación de Granada, de la que tenemos constancia de su funcionamiento en el año 1772, del que se conservan los libros de elecciones de cargos, habiendo sido destruido su archivo en una gran parte por las tropas de Napoleón que incendiaron la sede de dicha Academia con toda su documentación, y, no obstante, vandálica intervención se conservan documentos acreditativos de que esta Real Academia funcionaba ya en el primer tercio del siglo XVIII. 
A lo largo del siglo XVIII y del XIX se va produciendo la creación de otras Academias en Andalucía hasta que, a mediados del siglo actual, podemos reseñar la existencia de 17 Academias, distinguiendo básicamente las Academias especializadas, como son las de Bellas Artes, Medicina o Jurisprudencia y Legislación, de aquellas otras que tienen un caracter generalista y comprenden diversos sectores de las Ciencias, las Bellas Letras, las Nobles Artes, etc.

lgualmente es destacable la diversidad de Academias desde el punto de vista del territorio que abarca su jurisdicción, como son las de caracter provincial de Bellas Artes; de carácter regional como son las de Medicina; o de caracter estrictamente local que realizan su actividad en un solo municipio, o el supuesto más curioso de aquellas otras que tienen ámbito supranacional como es el caso de la Real Academia Hispanoamericana de Cádiz o la Iberoamericana de Farmacia.

\section{El regimen administrativo de las Academias andaluzas con anterioridad a la vigencia de la constitución}

Las Academias andaluzas alcanzaron el número de 16 antes de la vigencia de la Constitución de 1978, y que fueron las siguientes:

- Real Academia Provincial de Bellas Artes de Cádiz.

- Real Academia Hispano-Americana de Ciencias, Letras y Artes de Cádiz.

- Real Academia de Medicina y Cirugía de Cádiz.

- Academia de S. Dionisio, de Ciencias, de Artes y Letras de Jerez de la Frontera.

- Academia de S. Romualdo, de Ciencias, Artes y Letras de S. Fernando.

- Real Academia de Córdoba, de Ciencias, Bellas Letras y Nobles Artes.

- Real Academia de Bellas Artes de Nuestra Sra. de las Angustias de Granada.

- Real Academia de Jurisprudencia y Legislación de Granada.

- Real Academia de Medicina de Granada.

- Academia de Ciencias Matemáticas, Físico-Químicas y Naturales de Granada.

- Academia de Veterinaria de Andalucía Oriental, Granada.

- Real Academia de Medicina de Sevilla.

- Real Academia Sevillana de Buenas Letras. 
- Real Academia de Bellas Artes de Sta. Isabel de Hungria, de Sevilla.

- Academia de Ciencias Veterinarias de Sevilla.

- Academia de Bellas Artes y Buenas Letras Luis Vélez de Guevara, de Ecija.

- Real Academia de Bellas Artes de S. Telmo, de Málaga.

Las relacionadas Academias se regían por sus respectivos Estatutos, algunos de ellos vigentes desde los siglos XVIII y XIX, y que fueron, en algunas ocasiones, actualizados por la Administración central que ejercía la tutela de las mencionadas Academias, encontrándose adscritas, a los efectos de tutela y obtención de medios económicos por via de subvención, al Ramo ministerial que tenia a su cargo la Educación, y bajo las sucesivas denominación de "Instrucción Pública», "Educación Nacional", "Educación», "Educación y Ciencia», etc.

Las relaciones con la Administración central fueron siempre lejanas, distantes y frias, pues las mismas se reducian a la limitada función de aprobar las modificaciones estatutarias, que de una parte no eran muy numerosas, y de otra la fijación de modestas subvenciones, que escasamente llegaban a cubrir los más elementales gastos de funcionamiento.

Puede afirmarse que ha existido una invisible tensión entre las Academias Centrales y las Periféricas, generándose una incomunicación histórica entre ellas, salvo contadas excepciones, y destacando la importante labor que recientemente viene realizando el Instituto de España para relacionar, y de alguna forma integrar en su seno, todas las Academias de España.

A partir de la vigencia de la Constitución Española de 1978, se va a producir un importante cambio, ya que el fenómeno de descentralización autonómico alcanzará tambien a las Academias Andaluzas.

\section{Las Academias en la Constitución Española y en el Estatuto de Autonomía de Andalucía}

El Estauto de Autonomía de Andalucía establece la competencia exclusiva sobre las materias contenidas en el art. 13 del mismo, incluyendo dentro de dicha competencia exclusiva, en su apartado 29, la Investigación y sus Instituciones así como «las Academias con sede central en Andalucía", sin perjuicio de la remisión que este precepto hace al art. 149.1.15 de la Constitución que reserva al Estado, como competencia exclusiva, el fomento y coordinación general de la investigación científica y técnica. 
Cabe destacar que este sector competencial del art. 13 del Estatuto de Andalucía contempla en sus apartados 26 al 29 inclusive, un conjunto de aspectos relacionados con la Cultura y la protección del Patrimonio Histórico Artístico, Archivos, Museos, Bibliotecas, Conservatorios y Centros de Bellas Artes de interés para la Comunidad Autónoma, además de la remisión antes aludida, respecto a la competencia exclusiva sobre Investigación y sus Instituciones.

Procede indicar brevemente en este momento que la Constitución española de 1978 no hace referencia a lo largo de su articulado a las Reales Academias, desde el punto de vista competencial y de forma especial en el art. 148 que contempla la lista de competencias que pueden asumir las Comunidades Autónomas, a no ser que se integre bajo el rótulo de "fomento de la cultura y de la investigación" a que se refiere el citado art. 148.1.17, pero que, insistimos, en ningún momento hace mención específica de las Academias.

Tampoco las Academias aparecen como competencia exclusiva del Estado en la enumeración que se contiene en el art. 149, sin perjuicio de la posibilidad que deja abierta el párrafo $3^{\circ}$ del art. 149 en el sentido de que «las materias no atribuidas expresamente al Estado por esta Constitución podrán corresponder a las Comunidades Autónomas, en virtud de sus respectivos estatutos".

Este es el supuesto que asume el Estatuto de Autonomía de Andalucía cuando declara la competencia exclusiva de la Comunidad Autónoma sobre las "Academias con sede central en Andalucía", si bien se debe hacer la observación de que esta Comunidad Autónoma asumirá la competencia en función de la sede de la Academia, de tal manera que deja abierta la posibilidad de que la misma se extienda a Academias con domicilio o sede central en Andalucía y con actividad fuera de la Comunidad Autónoma, es decir, con actividad en otras Comunidades Autónomas e incluso Academias que tienen actividad supranacional.

En resumen la Comunidad Autónoma de Andalucía asumió competencia exclusiva sobre las Academias con sede central en Andalucía tras la vigencia del Estatuto andaluz aprobado por Ley Orgánica 6/1981, de 30 de diciembre, publicado en el BOE de 1 de enero de $1982, \mathrm{n}^{\circ} 9$.

La Constitución Española sólo contiene una sola referencia a las Academias al disponer en su art. 62.j), que corresponde al Rey «El Alto Patronazgo de las Reales Academias", cuyo mandato supone:

- Reconocer la tradicional vinculación de las Academias con la Corona, y el interés de la misma por estas tradicionales Instituciones Culturales. 
- Que este Alto Patronazgo entendemos que se extiende - por mandato constitucional- a todas las Reales Academias espanolas, sea cual fuere su localización geográfica o la especialidad científica o cultural de las mismas.

En la fase preautonómica se habían producido traspasos de funciones y servicios en materia de cultura a la Junta de Andalucía, entre las que pueden citarse el Real Decreto 1075/1981, de 24 de abril, BOJA $\mathrm{n}^{0} 13$ de 30 de junio, y el R.D. 1.124/1984, de 29 de febrero, BOJA $\mathrm{n}^{\circ} 83$ de 7 de septiembre, si bien los traspasos globales en materia de cultura de mayor importancia se producirán varios años despues en los RR.DD. $\mathrm{n}^{\circ}$ 864/1984, de 29 de febrero, que tiene un sentido genérico y completa las transferencias producidas en la fase preautonómica y el complementario R.D. 2764/1986, de 30 de diciembre, sobre modificación de medios adscritos a los servicios traspasados en materia de cultura.

De conformidad con las normas mencionadas la Comunidad Autónoma de Andalucía asumió las oportunas competencias de tutela administrativa sobre las Academias con sede central en Andalucía, y se fijó el correspondiente montante económico que comprendia el importe de las subvenciones abonadas por el Ministerio de Educación a las distintas Academias Andaluzas, y en ejecución de las normas mencionadas se produjo la adscripción administrativa de las Academia a la Consejería de Educación y Ciencia.

\section{El sentido coordinador de las Academias andaluzas}

\subsection{Iniciación de las actuaciones coordinadoras}

Las Academias Andaluzas comenzaron a relacionarse entre sí, de forma sectorial y en función de sus especialidades, con relativa antiguedad, y en especial teniendo en cuenta los intereses y materias comunes que ocupaban y preocupaban a las numerosas Academias andaluzas.

Tras la vigencia de la Constitución, los Académicos andaluces fueron conscientes de los cambios que podían generarse por la aplicación y desarrollo del Estatuto de Andalucía, por lo que en el año 1979, y durante los dias 8 al 11 de noviembre se celebró en Granada, y bajo los auspicios del Instituto de España, un Congreso de todas las Academias Andaluzas, celebrándose diversas sesiones de trabajo, conciertos, 
exposiciones, visitas, conferencias, etc, coincidiendo todos los asistentes en la necesidad de construir un lazo de unión entre todas las entidades, a cuyo efecto se convocó un segundo Congreso en Córdoba en el año 1981, y un tercero en Cádiz en 1983, donde cristalizaron importantes ideas de relación marcándose básicamente los siguientes objetivos:

- Conocimiento más amplio y profundo de la cultura andaluza (fuentes, raices, peculiaridades, etc) en todos los campos del saber.

- La intercomunicación de proyectos de investigación científica en las diferentes fases de preparación, realización, verificación de resultados, etc) entre las distintas Academias.

- Establecer una corriente de relaciones personales que lleve a un mutuo conocimiento de las Academias y de sus miembros.

- Analizar la problemática común a las Academias Andaluzas y establecer las bases para su resolución.

- Concienciar a la sociedad en general y a las distintas Autoridades en concreto, de la importante labor cultural y de investigación que las Academias vienen realizando en nuestro ámbito geográfico.

La sesión de trabajo del dia 21 de noviembre de 1981, en Cordoba, fué de una gran importancia ya que se aprobó la propuesta de creación de un Instituto de Reales Academias de Andalucía que coordinara a todas las Entidades académicas andaluzas teniendo como misión básica la de mantener la relación y coordinación entre las distintas Corporaciones andaluzas; preparar los correspondientes congresos bienales; elegir su Junta Rectora; promover el establecimiento de nuevas Academias en Andalucía para la realización de fines no comprendidos en el ámbito de las actuales Academias; publicar las Actas de los Congresos y reuniones así como los trabajos presentados a los mismos; y realizar cuantos trabajos conduzcan al más adecuado conocimiento científico y tradicional de Andalucía.

En consecuencia se consideró la constitución de dicho Instituto y su integración en el Instituto de España a cuyo efecto se creó una Comisión Coordinadora que se constituyó como "Consejo Coordinador de las Reales Academias de Andalucía" en sesión celebrada en dicha ciudad de Córdoba el 26 de Junio de 1982.

\subsection{Las fórmulas legales para la coordinación}

Se estudiaron diversas formulas asociativas, por medio de las cuales pudieran integrarse las Academias Andaluzas, llegándose a la conclusión 
que la solución ideal era la Corporación de Derecho Público, creada por Ley del Parlamento de Andalucía al que correspondía en exclusiva dicha potestad normativa, para lo cual se tuvo presente que las Corporaciones de Derecho Público tenían una larga trayectoria histórica que partía de los "Collegia" romanos y de los "Gremios" medievales, con toda la problemática monopolística que ello supuso, y su posterior colisión con el Estado Absoluto, que en Francia dió lugar al Edicto Turgot de 1776, y poco despues a la Ley Le Chapellier de 1791, que prohibió las Asociaciones y Corporaciones, y cuyo espíritu llega a la Constitución de Cádiz de 1810, y a la normativa dictada por aquellas Cortes Generales y Extraordinarias en defensa de la libertad de industria y del ejercicio profesional, como fueron los Decretos de 3 de Junio de 1813 y de 3 de Junio de 1823, llegándose a la total prohibición de Corporaciones y Asociaciones por el Decreto de 21 de octubre de 1820.

Sin embargo tal prohibición fué efímera, y a lo largo del siglo XIX se produce el renacimiento de las Corporaciones profesionales, que controla severamente el Estado autoritario del siglo actual, como sustitutivo del sindicalismo plural y de caracter diversificado, que de otra parte se trata de sustituir por sindicalismos unitarios en los sistemas nacional-sindicalista alemán, fascista-italiano o el verticalismo español.

Así pues podemos destacar el exacervado individualismo de la Revolución Francesa que rompe con todas las estructuras corporativas, y la posterior corporativización de los sistemas sindicales de los regímenes autoritarios del siglo $\mathrm{XX}$.

La Constitución de 1.978 superó, de una parte las técnicas individualistas de la Revolución Francesa, y estructura y reconoce una serie de sistemas asociativos, partiendo de la libertad de asociación del art. 22 de la Constitución, la constitucionalización de los Colegios profesionales, y las Organizaciones económicas a que se refiere el art. 52.

El desarrollo de estos principios constitucionales se produce en el art. 15 de la Ley del Proceso Autonómico, que se refiere de forma limitada a las Corporaciones de Derecho Público representativas de intereses económicos (entre las que se incluyen las Cámaras y Cofradias de Pescadores), y las Corporaciones de Derecho Público representativas de intereses profesionales, con clara remisión a los Colegios Profesionales, si bien reserva a la Ley formal la creación de Consejos Generales o Superiores de los distintos Colegios Profesionales.

Una parte de la doctrina viene insistiendo tambien en completar el esquema de la Administración Corporativa integrando en la misma 


\section{El Instituto de Academias de Andalucía}

las Federaciones Deportivas a partir de la Ley de Educación Física y el Deporte de 31 de marzo de 1980.

Hasta aquí el esquema tradicional de la Administración Corporativa española, respecto de la que debemos destacar el ámbito marginal en que se sitúa, entre el Derecho Público y Privado, pues generalmente se trata de asociaciones forzosas de particulares, a las que dota de personalidad el Estado, que desempeñan funciones de interés general, para lo que se les atribuyen determinados poderes públicos a la vez que gestionan intereses de caraccter particular de sus componentes.

Como se ve, en este esquema tradicional no cabe incluir ni a las Academias, en general, ni a la Institución que las coordinase, siendo extrapolable este planteamiento a todas las Academias existentes en España, ya tuviesen carácter Nacional, Regional, Provincial o Local, pues a lo largo de sus existencia no han sufrido los avatares, antes reseñados, por las Corporaciones y Asociaciones del siglo XIX, sino que por el contrario las Academias, como se ha indicado, tienen una pujante vida a través del siglo XIX, como Corporaciones de Derecho Público, e incluso para los constituyentes de Cádiz de 1810 no hubo la más mínima duda sobre su conveniencia, e incluso es destacable la promoción que de las mismas se produce a todo lo largo del siglo, así como en el actual sin que les afecten las limitaciones de los periodos autoritarios, salvo mínimos aspectos, ya que se respeta en gran medida para estas Corporaciones su estructura y funcionamiento democrático, y van a adquirir una especial dimensión en el Estado Social y Democrático de Derecho que configura la Constitución española de 1978, con especial tutela de cuanto suponga expansión y promoción de la cultura y de la investigación.

Entendemos que las Reales Academias en general, constituyen un "tertius genus" como Corporaciones de Derecho Público de carácter específico, dentro del más amplio ámbito de las Corporaciones de Derecho Público, atendiendo a los fines de interés general que satisfacen: Promoción y difusión de la cultura e investigación en general.

Conviene insistir en que la Cultura es la columna que vertebra la identidad de los pueblos, superando planteamientos sociológicos de antropología social o cultural, y haciendo otro de carácter universalista al integrar todos los aspectos de la sociedad y la cultura: Arte, literatura, lenguaje, religión, filosofía, ciencia, derecho, política, economía, organización social, urbanismo, ordenación del territorio, medio ambiente, etc., ya que la cultura es la forma más importante de la expresión, y de la concreción de la racionalidad humana, que demanda la intervención 
del poder político como tutelador de los valores fundamentales del individuo, del grupo social y de la sociedad organizada políticamente, para promover, promocionar y tutelar las instituciones consagradas a lo largo de varios cientos de años.

A partir de las anteriores ideas, se inició la labor del "Consejo Coordinador de las Reales Academias de Andalucía", conectando con la Junta de Andalucía y realizando una serie de estudios previos a los que se hace referencia seguidamente.

\section{El Consejo Coordinador de las Reales Academias de Andalucía}

Constituido el Consejo Coordinador, como antes se ha indicado, comenzó sus cambios de impresiones con las distintas Academias, así como con los Organos representativos de la Junta de Andalucía, a cuyo efecto se giraron visitas a las más altas instancias de la Administración andaluza, que acogieron el Proyecto con un gran interés, estimando la conveniencia y necesidad de estructurar un Organo que coordinara las distintas Academias con sede central en Andalucía.

Se creó una Comisión Permanente dentro del Consejo coordinador de las Reales Academias, ya que el Pleno estaba constituido por el Presidente, o representante, de las 17 Reales Academias, siendo necesaria la existencia de un órgano más reducido que pudiera reunirse y trabajar con mayor agilidad y flexibilidad.

Con base en estas ideas se crea la Comisión Permanente del Consejo Coordinador, a la que antes se hacia mención, compuesta por un Presidente, un Vicepresidente, un Vocal y un Secretario, que iniciaron seguidamente los estudios oportunos para preparar un Anteproyecto de Estatutos que regularían el Consejo coordinador de las Academias andaluzas.

Los estudios se centraron sobre los siguientes puntos básicos:

- Rango legal que tendría la Norma creadora del Instituto y naturaleza jurídica del mismo.

- Objeto del Instituto, tanto desde el punto de vista de las Academias, como en relación con la Administración pública de Andalucía.

- Estructura y organización del Instituto.

- Desarrollo estatutario de la norma de creación.

De forma sucinta se examinará a continuación cada uno de los apartados a que se ha hecho referencia, y que constituyen el esquema básico de la normativa reguladora del futuro Instituto. 


\subsection{Rango legal del Estatuto y su naturaleza jurídica}

\subsubsection{Rango legal}

Desde el primer momento se planteó cual sería el rango de la norma a través de la cual había de crearse el Instituto, es decir mediante Ley formal del Parlamento andaluz o la posibilidad de su creación a través de Norma Reglamentaria emanada del Consejo de Gobierno de la Junta de Andalucía.

Se llegó a la conclusión de la necesidad de cobertura de Ley formal de la Comunidad autónoma teniendo en cuenta que se pretendía crear una personalidad jurídica nueva con el caracter de Corporación de Derecho Público, por cuya razón se entendía la necesidad de acudir a la Ley Formal, sin perjuicio de la importancia que el contenido de la Norma tendría al regular el objeto, fines, organización, etc., del Instituto, que se configuraba como Organo de la Administración con todas sus consecuencias.

\subsubsection{El Instituto como corporación}

Como se ha apuntado uno de los aspectos esenciales que habia de contemplar la Norma era el de la creación de una entidad que se integraría en la realidad administrativa como Corporación de Derecho Público, determinando cual sería la forma de integrar el Instituto, a cuyo efecto solo cabían dos posibilidades:

- La fórmula integradora a partir de los Académicos Numerarios integrantes de las distintas Reales Academias. En este aspecto se podía utilizar como precedente la fórmula de creación del Instituto de España por Decreto de 1 de enero de 1938 (BOE de 2 de enero), cuyo art. $1^{\circ}$ disponía: "Recibe el nombre de Instituto de España el conjunto de los Académicos Numerarios de las Reales Academias...".

- La segunda posibilidad que se consideró fué la de proponer la creación del Instituto como Corporación de Corporaciones, es decir, mediante la integración de las distintas Academias andaluzas, todas ellas dotadas de personalidad jurídica como Corporaciones de Derecho Público. Según se verá esta fué la fórmula aceptada por el legislador regional. 


\subsection{Objeto del Instituto}

Se ha indicado antes la necesidad de la creación de esta entidad a fin de coordinar y relacionar las distintas Academias andaluzas, lo cual planteaba la consideración de un doble objetivo del Instituto:

- Promover y estrechar las relaciones entre las Reales Academias de Andalucía y asumir su representación en determinados momentos.

- Regular las realaciones que el Instituto tendría en el futuro con la Administración andaluza y las competencias que asumiría en este ámbito relacional.

\subsection{Estructura y organización}

La Ley de creación del Instituto debería regular la organización y estructura del Instituto, para hacer eficaz el funcionamiento del mismo, a cuyo efecto se estimaban necesarios dos tipos de Organos:

- De carácter individual a cuyo cargo correría la dirección, gestión y administración del Instituto mediante la creación de diversos cargos unipersonales: Presidente, Vicepresidentes, Tesorero, Vocal.

- Otros Organos, de carácter colegiado, a los que correspondería la adopción de los acuerdos de mayor importancia para el funcionamiento de la entidad, distinguiendo el Pleno del Instituto y la Junta de Gobierno.

\subsection{Desarrollo estatutario}

La Ley de Creación del Instituto se concebía como una norma que, en un reducido articulado, comprendiera las lineas fundamentales expuestas con anterioridad. Como consecuencia sería necesario realizar el desarrollo reglamentario y más detallado de los distintos aspectos regulados básicamente en la Ley, lo cual supondría la redacción y aprobación de los correspondientes Estatutos, así como determinar la forma de redacción o elaboración de los mismos por los que habría de regirse en el futuro la entidad.

Con dicha finalidad podían adoptarse dos criterios distintos:

- Que la Ley autorizara al Gobierno andaluz para el desarrollo reglamentario de la misma. 
- Que el Instituto, tras su constitución, elaborara los Estatutos que serían aprobados, en definitiva, por el Organo competente de la Administración andaluza.

Como más adelante se indicará se eligió esta segunda fórmula.

\section{El proyecto de Estatutos de un Instituto de Reales Academias de Andalucía}

La Comisión Permanente procedió a redactar el oportuno borrador o Proyecto de estatutos que fué objeto de detenido estudio dentro de dicho Organo, para ser elevado más tarde al Pleno del Consejo Coordinador, que tambien realizó un detenido estudio de los distintos extremos, siendo aprobado el Proyecto en la reunión celebrada el 26 de febero de 1983 en Antequera (Málaga).

Los debates fueron extensos e intensos participando en ellos la totalidad de las Academias existentes en Andalucía en aquellas fechas, y una vez que se llegó al consenso sobre el texto que se estimó más adecuado, fué remitido a la Administración de la Comunidad Autónoma andaluza con cuyos Organos representativos se tuvieron distintas reuniones y cambios de impresiones, hasta que, tanto la Presidencia de la Junta, como la Consejería de Educación y Ciencia, manifestaron sus criterios, que coincidieron, en una gran medida con el Proyecto elaborado con el Consejo Coordinador de las Academias, como se puso de relieve en la reunión celebrada en la Consejería de Educación y Ciencia de la Junta de Andalucía el dia 9 de Noviembre de 1983, a la que asistieron, de una parte, los miembros de la Comisión Permanente del Consejo Coordinador y, de otra, los altos representantes de la Junta de Andalucía, y en cuya reunión se produjo el consenso en relación con el texto definitivo que se elevaría al Consejo de Gobierno de la Junta de Andalucía.

7. La Ley creadora del Instituto de Academias de Andalucía, Ley 7/1985 de 6 de diciembre (BOJA DE 14 de diciembre)

\subsection{El Proyecto De Ley y su tramitación parlamentaria}

Aprobado el Proyecto de Ley por el Consejo de Gobierno, éste lo remitió al Parlamento de Andalucía, siendo publicado en el Boletín Oficial del Parlamento de Andalucía, de 9 de octubre de 1985, remi- 
tiendose a la Comisión de Educación y Cultura con apertura del plazo para presentación de enmiendas.

Solamente se presentaron nueve enmiendas que no afectaban sustancialmente al Proyecto de Ley sino que por el contrario la adicionaban, aclaraban o complementaban, según se pudo apreciar en el consiguiente debate, siendo aprobado definitivamente el texto de la Ley 7/1985, de 6 de diciembre por la que se creó el Instituto de Academias de Andalucía (BOJA del 14 de diciembre).

Como antes se ha dicho es una Ley muy corta ya que está integrada por seis artículos, una Disposición Transitoria y otra Disposición Final.

La Disposición Transitoria disponía que en el plazo de un mes a partir de la publicación de la Ley, se formaría una Junta Constituyente integrada por los Presidentes de todas y cada una de las Academias que se encargaría de elaborar los Estatutos por los que se regiría el Instituto de Academias de Andalucía, los cuales deberían elevarse al Consejo de Gobierno para su aprobación.

\subsection{Los Estatutos del Instituto de Academias de Andalucía}

Dando cumplimiento a la Disposición Transitoria antes citada se reunió el Consejo Coordinador de las Reales Academias de Andalucía el 11 de enero de 1986, en Arcos de la Frontera (Cádiz), procediendose a la formación de la Junta Constituyente integrada por todos los Presidentes de las Academias Andaluzas, y aprobándose el Proyecto de Estatutos en la misma sesión, siendo elevados a la Consejería de Educación para su aprobación por el Consejo de Gobierno.

Cabe hacer similar observación respecto de los Estatutos a la realizada en relación con la Ley, ya que se trata de un texto conciso y claro compuesto por 16 artículos y dos Disposciones Transitorias, a través de cuyo articulado se desarrollan las lineas esenciales reguladas por la Ley de creación del Instituto.

El repetido texto fué aprobado por Decreto 265/1986, de 24 de septiembre, y publicado en el BOJA de 21 de octubre de 1986.

Con carácter complementario los Estatutos hacen remisión a las Normas de carácter interno que deberá aprobar el Instituto, como son los Reglamentos de: Régimen Interior, aprobado en 23 de enero de 1988, para regular el régimen de sesiones de los Organos de Gobierno y funcionamiento en general; el Reglamento de Honores y Distinciones aprobado en la misma fecha, y la aprobación del emblema e insignia 
del Instituto, Medalla y Distintivos del mismo, aprobado tambien en la misma sesión de 23 de enero de 1988.

\section{El Instituto de Academias en su normativa reguladora}

\subsection{Justificación legal}

Tanto el Preámbulo de la Ley 7/85, de 6 de diciembre, como el del Decreto 265/1986, de 24 de septiembre, ponen de manifiesto que la Junta de Andalucía tiene competencia exclusiva sobre las Academias con sede central en la misma, destacando que dichas Academias venian desarrollando actividades en los distintos campos del saber de forma aislada y esporádica siendo por ello necesario contar con un Organismo, como es el Instituto de Academias de Andalucía, "que las aúne y, en el ámbito de actuación propia de cada Academia preste su asesoramiento en las consultas que les plantee el Gobierno Andaluz" siendo manifiestos los méritos y prestigio de los Académicos andaluces, su gran tradición en los distintos campos de la cultura, la independencia de su posiciọn y gestión y la renovada vitalidad de sus actividades.

\subsection{El Instituto como Corporación de Derecho Público}

Se crea el Instituto de Academias de Andalucía como Corporación de Derecho Público, constituido por todas las Academias que tienen su sede central y realizan su actividad dentro del territorio de Andalucía, a cuyo efecto relaciona la Ley nominativamente las 17 Academias que lo integran, incorporándose al mismo aquellas Academias que se creen en el futuro siempre que sean Corporaciones de Derecho Público (art. 1 de la Ley y art. 1 de los Estatutos).

\subsection{Objeto del Instituto}

Se regula en un doble aspecto: Desde el punto de vista de las relaciones entre las Academias Andaluzas y respecto a la Junta de Andalucía.

En el primer aspecto, es objeto del Instituto mantener y estrechar las relaciones de todo tipo entre las Academias andaluzas, ostentando 


\section{Eduardo Roca Roca}

la representación académica del conjunto de todas ellas, realizando las oportunas funciones de coordinación "sin menoscabo de la autonomía propia de cada una de ellas", promoviendo el desarrollo de la cultura y la investigación en el más amplio sentido y, en especial, en materias que afectan a Andalucía.

El Instituto se relacionará con las Reales Academias españolas sea cual fuere su ámbito territorial así como con el Instituto de España y con las Administraciones del Estado, Autónoma y Local, pudiendo convocar toda clase de Congresos, reuniones, conferencias, etc. (art. 2 de la Ley y art. 2 de los Estatutos).

En relación con la Administración andaluza el Instituto "es Organo asesor y consultivo de la Junta de Andalucía, cuyos distintos Organos podrán recabar su parecer en asuntos que afecten al ámbirto de las distintas Academias andaluzas" (art. 3 de la Ley y art. 3 de los Estatutos).

Como consecuencia de dicho mandato legal el Instituto se encuentra representado en la actualidad en diversas Comisiones, Consejos, $\mathrm{Pa}$ tronatos y Fundaciones de Andalucía, como es el Consejo Consultivo de Andalucía o las Comisiones del Patrimonio Histórico.

Igualmente el Instituto debe informar con carácter preceptivo a la Administración Andaluza en los supuestos de creación de nuevas Academias que tengan el carácter de Corporaciones de Derecho Público, y en los casos de modificación de los Estatutos y Reglamentos de las Academias existentes (art. 4 de la Ley y art. 3.2. de los Estatutos).

\subsection{Organos de Gobierno}

El Instituto se gobierna a través de Organos unipersonales y colegiados.

- Son Organos unipersonales: el Presidente, dos Vicepresidentes, el Secretario General, el Tesorero y el Vocal, pudiendo recaer dichos cargos tanto en Presidentes o Directores de Academia o en Académicos Numerarios, si bien el Presidente y los dos Vicepresidentes serán nombrados por el Consejero de Educación y Ciencia de la Junta de Andalucía a propuesta del Pleno del Instituto. A estos cargos unipersonales les corresponden las funciones propias de presidencia, secretaría, gestión económica, etc., y tendrán una duración de cuatro años, renovándose por mitad cada dos años (arts. 6 al 11 de los Estatutos). 
- Son Organos Colegiados: el Pleno y la Junta de Gobierno. El Pleno está compuesto por los Presidentes o Directores de las Academias que integran el Instituto, así como por los cargos directivos integrantes de la Junta de Gobierno que no tuvieren la condición de Presidente. El Pleno tiene las competencias más importantes relacionadas con la actividad presupuestaria, elección de cargos directivos, aprobación de Reglamentos de Régimen Interior y de Honores y Distinciones.

La Junta de Gobierno ejerce las competencias que no estén atribuidas expresamente al Pleno y, en especial, el desarrollo y cumplimiento de los acuerdos adoptados por el Pleno, así como adoptar los acuerdos correspondientes en caso de urgencia (art. 6 de la Ley y arts. 13 y ss. de los Estatutos).

\subsection{Recursos económicos}

Para el cumplimiento de sus fines, el Instituto cuenta con los siguientes recursos económicos:

- Las subvenciones que pueda recibir de las Administraciones Públicas, y de cualquier otro ente $u$ organismo de naturaleza pública, que se justificarán en la forma establecida en la Normativa vigente.

- Toda clase de donaciones, herencias y legados.

- El producto y rendimiento de sus bienes, publicaciones y actividades.

Se entiende que el Instituto puede percibir cualquier tipo de subvención procedente de personas físicas o jurídicas de Derecho Privado, ya que la rúbrica general de "donaciones" comprende cualquier tipo de percepción económica que pueda recibir con carácter gratuito.

\section{Conclusión}

Los trece años de vida del Instituto de Academias de Andalucía ponen de manifiesto la conveniencia de su creación, ya que se están cumpliendo satisfactoriamente sus objetivos, pues existe una estrecha relación de las Academias que integran la Entidad, a cuyo efecto se celebran varias reuniones del Pleno a lo largo del Curso Académico, entre las que destacan la Apertura del Curso, el dia del Instituto y la Clausura del Curso Académico. Estas sesiones son públicas y se 


\section{Eduardo Roca Roca}

celebran, de forma rotativa, en diversas ciudades Andaluzas, a fin de que su actividad pueda ser conocida en todo el territorio de la Comunidad Autónoma. Asimismo celebra reuniones privadas, para tratar de los temas que son de la competencia del Pleno.

La Junta de Gobierno celebra reuniones periódicas para atender al funcionamiento ordinario del Instituto, o resolver los asuntos urgentes, dando cuenta al Pleno en su primera reunión.

Son fluidas las relaciones del Instituto con la Administración Andaluza, que requiere el consejo del mismo en diversas ocasiones, y también mantiene contacto con el Instituto de España, cuya presencia es constante en los principales actos del Instituto Andaluz, que tambien se relaciona con otras Instituciones Científicas y Culturales españolas y extranjeras. 